\title{
Erratum: Distinguishing a rotating Kiselev black hole from a naked singularity using the spin precession of a test gyroscope \\ [Phys. Rev. D 98, 024015 (2018)]
}

\author{
Muhammad Rizwan, Mubasher Jamil, and Anzhong Wang
}

(ब) (Received 19 June 2019; published 10 July 2019)

DOI: $10.1103 /$ PhysRevD.100.029902

There is an error in Eq. (3). The total mass and angular momentum should be given, respectively, by

$$
\begin{aligned}
M_{T} & =M+\frac{\alpha r^{-1-3 \omega}}{2 a}\left[a r+\left(r_{0}^{2}+a^{2}\right) \arctan \left(\frac{a}{r_{0}}\right)\right], \\
J_{T} & =j+\alpha r_{0}^{-3 \omega}\left[\frac{a(2-3 \omega)}{4}-\frac{3 \omega r_{0}^{2}}{4 a}+\frac{3 \omega\left(r_{0}^{2}+a^{2}\right)^{3}}{4 a^{2} r_{0}} \arctan \left(\frac{a}{r_{0}}\right)\right] .
\end{aligned}
$$

